\title{
Psycho-emotional disorders in women with unplanned pregnancies
}

\author{
Patricia Cortés-Salim ${ }^{1}$, Marisol González-Barrón ${ }^{2}$, Gustavo Romero-Gutiérrez ${ }^{1, ~ *}$ \\ ${ }^{1}$ Department of Obstetrics and Gynecology, Hospital de Gineco-Pediatría $N^{\circ}$ 48, León, México \\ ${ }^{2}$ Department of Clinical Psychology, Unidad Médica de Alta Especialidad, Hospital de Gineco-Pediatría No 48 León, Guanajuato, México
}

\section{Email address:}

pato114@hotmail.com (P. Cortés-Salim), aztlan8516@hotmail.com (M. González-Barrón), gustavoromerog@hotmail.com (G. Romero-Gutiérrez)

\section{To cite this article:}

Patricia Cortés-Salim, Marisol González-Barrón, Gustavo Romero-Gutiérrez. Psycho-Emotional Disorders in Women with Unplanned Pregnancies. American Journal of Health Research. Vol. 2, No. 1, 2014, pp. 27-32. doi: 10.11648/j.ajhr.20140201.15

\begin{abstract}
Introduction: Unplanned pregnancy has been associated with postpartum depression but there is little information about other psycho-emotional disorders. Objective: We aimed to determine the presence of psycho-emotional disorders in women with unplanned pregnancy. Methods: Six-hundred and four women were recruited and divided into two groups: One consisting of 302 women with unplanned pregnancies and one with 302 women with planned pregnancies. To evaluate the presence of psycho-emotional disorders, the Minnesota Multiphasic Personality Inventory-2 was applied. Results: Women with unplanned pregnancies had more psycho-emotional disorders $(66.2 \%$ vs. $43.7 \%$, respectively, p $<$ $0.001)$. The most common disorders were anxiety $(39.4 \%)$, post-traumatic stress $(37.1 \%)$ and depression $(31.5 \%), p=0.001$. Conclusion: Women with unplanned pregnancies have more frequency of psycho-emotional disorders. It is suggested screening not only for postpartum depression, but also for more comprehensive psycho-emotional disorders in women with unplanned pregnancies. Then early psychological support could be provided to the mother.
\end{abstract}

Keywords: Minnesota Multiphasic Personality Inventory-2, Unplanned Pregnancy, Psycho-Emotional Disorder

\section{Introduction}

Conventional measures of unintended pregnancy are designed to reflect a woman's intentions before she became pregnant. Unintended pregnancies are pregnancies that are reported to have been either unwanted (i.e., they occurred when no children, or no more children, were desired) or mistimed (i.e., they occurred earlier than desired). In contrast, pregnancies are described as intended if they are reported to have happened at the 'right time' or later than desired (because of infertility or difficulties in conceiving). A concept related to unintended pregnancy is unplanned pregnancy, one that occurred when the woman used a contraceptive method or when she did not desire to become pregnant but did not use a method [1].

Planned pregnancies occur when a couple or a woman decides to have a child, but can also occur in less stable situations that pose risks for the family and the child. Unplanned pregnancies may be welcomed or undesired, depending on the circumstances [2]. Unplanned pregnancies, especially among teenagers, can hamper the ability of young women to complete their education and effectively participate in the workforce. To determine whether and when to bear children is one of the most basic aspects of self-determination, and it has become a prerequisite for the full participation of women in modern society [3]. The worldwide prevalence of unplanned pregnancy is very high, for example in Britain (England, Scotland and Wales) is $29 \%$, and most unplanned pregnancies occur in women aged 20-34 years [4].

Some researchers have found association between unplanned pregnancy and postnatal depressive symptoms; however some socio-demographics factors such as, marital status, maternal age and education of mother, showed no significant association with postpartum depression [5].

In countries of Latin America, unplanned pregnancy is a reality for many young, poor women, and it can impact physical, emotional, socio-economic and family dimensions [6]. The Woman and Health Latin American Center presented a report on women's International Day 2008, stating that every minute in some place of the world, 300 women conceive without wishing or planning their pregnancy [7].

Some investigators described a link between maternal 
anxiety levels early in pregnancy and the child's susceptibility to attention deficit hyperactivity disorder years later. It is has also been described that stress and anxiety during pregnancy can increase the risk of miscarriage [8].

In a study carried out in Ankara, Turkey, the researchers found that in the postnatal period, maternal depressive symptoms were significantly associated with unplanned pregnancy, increased anxiety, and perceived lower satisfaction with paternal physical support [9].

There is little information regarding other psycho-emotional disorders that may occur in pregnant women with unplanned pregnancies. Our aim was to determine the presence of psycho-emotional disorders in these women at immediate postpartum.

\section{Methods}

\subsection{Design and Settings}

A cohort study was conducted at the Hospital of Obstetrics and Gynecology at the Mexican Institute of Social Security in Leon, Mexico. Six-hundred twenty-two women with a eutocic delivery resulting in the birth of a healthy newborn were invited to participate; the recruitment was carried out from the patients who were hospitalized; and with a computer program of random numbers, they were progressively included until the sample was completed. Women were invited to participate at 1-2 days after delivery; this date was assigned conventionally because at our hospital postpartum women are discharged during this time.

The study protocol was approved by the ethics and research committee of the local institutional review board, and the women who agreed to participate in the study completed a written informed consent form. The recruited women answered face-to-face questions from the Minnesota Multiphasic Personality Inventory-2 (MMPI-2) administered 1-2 days after delivery in a Spanish language adapted by researchers PCS and MGB.

The MMPI-2 Spanish version has 567 items, all true-or-false questions, and usually takes between 60 and 90 minutes to complete. The MMPI-2 is a recent version of the original MMPI that was designed to help identify personal, social, and behavioral problems in psychiatric patients. This MMPI-2 edition can be used in healthy individuals [10]; and this questionnaire recently has been validated in a population of pregnant women [11]. According to responses of women 17 psycho-emotional disorders can be categorized by reaching a specific number of positive answers.

\subsection{Study Participants}

Women were divided into 2 groups: Three-hundred and two women who had an unplanned pregnancy and three-hundred and two women who had a planned pregnancy. This division according to planned/unplanned is unspecific, but we thought was the only manner to determine the presence of any difference between the groups on this condition; the women were matched by weeks of gestation and their referral status.

Because of possible participation of regional characteristics, we evaluated some socio-demographic variables of our population as well as clinical factors; the factors registered were: maternal age, which was defined as completed years at the time of delivery; marital status, which was registered as 0 if the mother was currently married and as 1 if the mother had never married or was divorced, separated, or widowed (single women); education level, which was registered as the number of completed years of school; maternal occupation, which was categorized as 0 if the woman had no formal occupation and as 1 if she had employment outside the home; parity, which was defined as the number of previous births, including stillbirths; previous pregnancies, including abortions; we asked the participants to describe their pregnancy as planned vs. unplanned, which was categorized as 0 for planned (when a couple or a woman decides to have a child), and 1 for unplanned (the occurrence of a pregnancy even when a couple or a woman did not decide to have a child); we did not consider if the unplanned pregnancies were either welcomed or undesired; we also registered gestational age at the moment of delivery; number of dead sons; antenatal care, which was categorized as 0 when the woman received no antenatal care at the hospital and 1 when she attended at least one appointment in the antenatal care department; the number of antenatal visits for each woman, coded as the current number of prenatal appointments during the pregnancy; acceptance of a contraceptive method after the present pregnancy; and the following variables were registered conventionally as yes $=1$ or not $=0$, according to responses of women about: enough and accurate medical information during antenatal care; family support during pregnancy; and interfamilial violence. Also clinical factors as medical conditions and obstetric complications were included; and were coded as 1 when present and 0 when absent.

During the study period, 622 postpartum women were invited to take the MMPI-2; six hundred and four women agreed to participate and eighteen did not, corresponding to a response rate of $97.1 \%$

\subsection{Data Analysis}

Statistical analysis included percentages, mean and standard error, student's-test or Mann-Whitney U test for continuous variables as appropriate depending on their normal distribution, and chi-square or Fisher Exact' test for categorical variables. To determine which socio-demographic and clinical variables were associated with psycho-emotional disorder, a logistic regression analysis was carried out. Both socio-demographic and clinical factors were considered as regressors (predictive variables). The presence $=1$, or absence $=0$ of psycho-emotional disorder, was considered as the outcome 
measure.

The alpha value was set at 0.05 . Odds ratios (OR) with 95\% confidence intervals (CIs) were estimated. The statistical analysis was performed with Number Cruncher Statistical System software (Number Cruncher Statistical System Inc., Kaysville, UT) [12].

\section{Results}

In the entire sample, the maternal age averaged $26.2 \pm$ 0.2 years. The average number of school years was $8.7 \pm$ 0.1 . Five hundred and forty-five women were married (90.2\%). Three hundred fifty-eight women (59.3\%) did not work outside home and two-hundred forty-six women (40.7\%) worked outside home. The number of pregnancies averaged 2.4. The average gestation age was $38.6 \pm 0.1$ weeks. A total of 61 women $(10.1 \%)$ were teenagers, between 14 and 19 years old. Among these teenager women there were 27 women $(44.2 \%)$ with planned pregnancies and $34(55.8 \%)$ with unplanned pregnancies.

In the univariate analysis of socio-demographic backgrounds two factors reached statistical significance: low educational level, $\mathrm{p}=0.033$, and single women, $\mathrm{p}<$ 0.001, (table 1).

In the analysis of the clinical characteristics there were 5 significant factors: number of previous pregnancies, $\mathrm{p}<$ 0.001 ; number of antenatal visits, $\mathrm{p}<0.001$; medical advice, $\mathrm{p}=0.003$; contraceptive acceptance after delivery, $\mathrm{p}=$ 0.036 and psycho-emotional disorders, $\mathrm{p}=0.001$, (table 2 ).

Table 1. Univariate analysis of socio-demographic backgrounds of the two groups [mean $\pm S E ; n(\%)]$.

\begin{tabular}{|c|c|c|c|}
\hline Variable & Women with unplanned pregnancies $(n=302)$ & Women with planned pregnancies $(\mathrm{n}=\mathbf{3 0 2})$ & $\mathbf{P}$ \\
\hline Maternal age & $26.1 \pm 0.3$ & $26.2 \pm 0.3$ & 0.752 \\
\hline Education level & $8.5 \pm 0.1$ & $9.0 \pm 0.1$ & 0.033 \\
\hline Marital status (single women) & $43(14.2)$ & $16(5.2)$ & 0.001 \\
\hline Family support & $291(96.4)$ & $292(96.7)$ & 1.000 \\
\hline Working women & $116(38.4)$ & $130(43.0)$ & 0.282 \\
\hline
\end{tabular}

Table 2. Univariate analysis of clinical factors between the groups [mean $\pm S E ; n(\%)]$.

\begin{tabular}{|c|c|c|c|}
\hline Variable & $\begin{array}{l}\text { Women with unplanned pregnancies } \\
\qquad(\mathrm{n}=\mathbf{3 0 2})\end{array}$ & $\begin{array}{l}\text { Women with planned } \\
\text { pregnancies }(\mathrm{n}=302)\end{array}$ & $\mathbf{P}$ \\
\hline Previous pregnancies & 2.6 & 2.7 & 0.001 \\
\hline Dead sons & 3.3 & 3.6 & 0.832 \\
\hline Number of antenatal visits & 7.9 & 8.8 & 0.001 \\
\hline Obstetric complications & 0.2 & 0.2 & 0.457 \\
\hline Psycho-emotional disorder & 2.9 & 1.5 & 0.001 \\
\hline Tobacco abuse & $17(5.6)$ & $17(5.6)$ & 0.860 \\
\hline Interfamilial violence & $26(8.6)$ & $20(6.6)$ & 0.443 \\
\hline Medical advice & $273(90.4)$ & $292(96.7)$ & 0.003 \\
\hline Contraceptive acceptance (after this pregnancy) & $257(85.1)$ & $236(78.1)$ & 0.036 \\
\hline
\end{tabular}

Table 3. Logistic regression analysis to determine which scio-demographic and clinical variables are associated with psycho-emotional disorder ( $n=604)$.

\begin{tabular}{|c|c|c|c|c|}
\hline Variable & Regression coefficient & $\mathbf{P}$ & Odds ratio & $(95 \%)$ Confidence intervals \\
\hline Maternal age & -4.209 & 0.983 & 0.59 & $0.25-1.43$ \\
\hline Single women & 0.080 & 0.013 & 2.24 & $1.18-4.25$ \\
\hline Education level & -0.187 & 0.001 & 2.03 & $1.40-2.94$ \\
\hline Previous pregnancies & 0.009 & 0.906 & 1.15 & $0.78-1.71$ \\
\hline Number of antenatal visits & 4.737 & 0.091 & 6.35 & $0.59-67.5$ \\
\hline Obstetric complications & -6.670 & 0.973 & 0.97 & $0.66-1.43$ \\
\hline \multicolumn{5}{|l|}{ Model summary: } \\
\hline Model & Model & Model & Mode 1 & \\
\hline R-squared & Degree freedom & chi-square & $\mathrm{P}$ & \\
\hline 0.112990 & 7 & 75.9 & 0.001 & \\
\hline
\end{tabular}

After logistic regression analysis, only three factors remained significant: single women, $\mathrm{p}=0.013,(\mathrm{OR}=2.24$, 95\% CI: 1.18-4.25); low education level, $\mathrm{p}=0.001,(\mathrm{OR}=$ 2.03, 95\% CI: 1.40-2.94); and unplanned pregnancy, $\mathrm{p}=$
$0.001,(\mathrm{OR}=2.23,95 \% \mathrm{CI}: 1.59-3.14)$.

As we observed, other factors such as previous pregnancies, education level, number of antenatal visits, and marital status lost their significance (table 3 ). 
Of the 302 women with unplanned pregnancies, 200 (66.2\%) had a psycho-emotional disorder, whereas in women with planned pregnancies, 132 women $(43.7 \%)$ had at least one disorder, $\mathrm{p}<0.001$. The most common disorders in both groups were anxiety, observed in 170 women $(28.1 \%)$, post-traumatic stress in $164(27.2 \%)$, and depression in $146(24.2 \%)$. For women with unplanned pregnancy, the most common disorder was anxiety, observed in 119 patients (39.4\%), followed by post-traumatic stress in 112 patients $(37.1 \%)$ and depression in 95 patients $(31.5 \%)$. In women with planned pregnancies, post-traumatic stress was the most common disorder, observed in 52 women $(17.2 \%)$; anxiety and depression each appeared in 51 women $(16.9 \%)$, (table 4$)$.

Table 4. Comparison of the psycho-emotional disorders between unplanned and planned pregnancies, $n(\%)$.

\begin{tabular}{|c|c|c|c|}
\hline $\begin{array}{l}\text { Psycho-emotional } \\
\text { disorder }\end{array}$ & $\begin{array}{l}\text { Women with } \\
\text { unplanned } \\
\text { pregnancies } \\
(\mathbf{n}=\mathbf{3 0 2}) \\
\end{array}$ & $\begin{array}{c}\text { Women with } \\
\text { planned } \\
\text { pregnancies } \\
\mathbf{n = 3 0 2 )} \\
\end{array}$ & $\mathbf{P}$ \\
\hline Anxiety & $119(39.4)$ & $51(16.9)$ & 0.001 \\
\hline Post-traumatic stress & $112(37.1)$ & $52(17.2)$ & 0.001 \\
\hline Depression & $95(31.5)$ & $51(16.9)$ & 0.001 \\
\hline Low self-esteem & $74(24.5)$ & $37(12.3)$ & 0.001 \\
\hline Family disaffection & $74(24.5)$ & $40(13.2)$ & 0.001 \\
\hline Schizophrenia & $72(23.8)$ & $38(12.6)$ & 0.001 \\
\hline Psychasthenia & $58(19.2)$ & $32(10.6)$ & 0.004 \\
\hline Fears & $56(18.5)$ & $21(7.0)$ & 0.001 \\
\hline Anger & $50(16.6)$ & $26(8.6)$ & 0.005 \\
\hline Psychopathic deviate & $48(15.9)$ & $31(10.3)$ & 0.054 \\
\hline Sadness & $36(11.9)$ & $23(7.6)$ & 0.100 \\
\hline Hypomania & $34(11.3)$ & $37(12.3)$ & 0.801 \\
\hline Paranoia & $31(10.3)$ & $18(6.0)$ & 0.074 \\
\hline Social introversion & $26(8.6)$ & $16(5.3)$ & 0.150 \\
\hline Hypochondriasis & $4(1.3)$ & $0(0)$ & 0.132 \\
\hline Conversion hysteria & $2(0.7)$ & $0(0)$ & 0.479 \\
\hline $\begin{array}{l}\text { Femininity role } \\
\text { rejection }\end{array}$ & $1(0.3)$ & $0(0)$ & 1.000 \\
\hline
\end{tabular}

\section{Discussion}

The news of pregnancy can be received with mixed feelings from happiness to insecurity, fear, or even initial rejection. Almost every woman will have a degree of ambivalence as to whether they want the baby. In $30 \%$ of cases, the pregnancy was not planned and may have occurred at an inopportune moment [13]. In our study, fear appeared in $18.5 \%$ of women with unplanned pregnancies, and insecurity was evaluated as low self-esteem, which appeared in $24.5 \%$ of patients.

It has been reported an association between unplanned pregnancies and being single or divorced; similarly, we found more single women in the group with unplanned pregnancies. Recently, also it has been described association between poor marital relationships with peripartum depression [14].
In sexual encounters, women with unplanned pregnancies were more likely to use no contraception or to practice "withdrawal" or use condoms rather than hormonal contraception [15]. In our study population, we found that contraceptive methods were more accepted in women with unplanned pregnancies than in those with planned pregnancies.

Low educational status has been associated with more than double the risk of unplanned pregnancy at one year after delivery [16], similarly, we also found low education levels in women with unplanned pregnancies. Others investigators have reported that women identified as victims of physical violence were more likely to report an unplanned pregnancy than those who were not victims [17]. Similarly, in our findings, more women with unplanned pregnancies suffered from violence.

In a study carried out in an African population, it was evaluated postpartum depression, using the twenty five-item Self-Reporting Questionnaire; and major depression was confirmed using the Mini International Neuro-Psychiatric Interview; potential associations were analyzed between major depression and the respondents' demographics and various psychological, social and obstetric factors; psychiatric disorder was significantly associated with young age, being single, negative life events, unplanned pregnancy, unwanted sex of baby and current physical illness in both mother and newborn [18]. These findings are similar to those we found in our study using the MMPI-2.

In other research, it was used the Edinburgh Postnatal Depression Scale at two sampling time points as well as the Structured Clinical Interview for the DSM-III-R, to determine that psychosocial risk factors are associated with postnatal depression; the identified psychosocial factors were: 16 years old or younger, past history of psychiatric illness, experiencing one or more life events, marital dissatisfaction, experiencing unsatisfactory social support, a vulnerable personality and having a baby of undesired sex [19]. In our study, 3.4\% of women reported unsatisfactory family support and $6.4 \%$ unsatisfactory medical information; these factors were most common in women with unplanned pregnancies.

These previous studies have documented a link between parental depression and a greater risk of behavioral problems as well as cognitive deficits (poor problem-solving skills, impaired school performance, and intellectual acuity) in the children when compared to children of non-depressed parents. However, we found, that unplanned pregnancy is an important risk factor not only for postpartum depression, but also for other psychological disorders such as anxiety, post-traumatic stress and low self-esteem, as similarly it has been recently reported [20].

There are some limitations to our study. The design of the study is weak because it is important to control for psychopathology before pregnancy and other confounding effects. Therefore, the description of psycho-emotional disorders in pregnancy requires a longitudinal prospective 
design; moreover, ours was a hospital-based study, and for that reason the results cannot be generalized to the total population. The application of the questionnaire was done at immediate postpartum and the answers might have been influenced by state of the mother with respect to complications that developed during pregnancy and delivery. Also it is possible recall bias, because some mental health issues develop with time and the limited follow up in our study may not be adequate for identifying significant problems.

Although the MMPI-2 is a clinical testing instrument that has been internationally validated and is considered the most comprehensive index for detecting personality and emotional disorders, it is complex and time-consuming, and should be used in conjunction with other assessment tools. Diagnosis should never be made solely on the results of the MMPI-2 test. For example, some disorders which appeared associated to unplanned pregnancy, such as schizophrenia, need a specific psychological test in order to confirm the diagnosis of this disease.

Despite these drawbacks, our findings could be useful for these reasons: First, there is little literature about reproductive impact of unplanned pregnancies in developing countries settings. Second, despite the hospital-based design of our research, it allowed us to find significant association between socio-demographic and clinical factors with unplanned pregnancies. Third, thanks to this study we found a surprisingly high frequency of psycho-emotional disorders in women with unplanned pregnancies at immediate postpartum $(66.2 \%)$.

This is a new and the more important finding in our study. Because in a similar study [21], the authors investigated only depression associated with unplanned pregnancies at 10 days postpartum and found that $48.7 \%$ of these women experienced depression. They, in turn, recommended that women with unplanned pregnancies should be screened for depression.

Supported by our findings, we suggest screening not only for depression but also for more comprehensive psycho-emotional disorders that could be identified earlier such as screening to be done during pregnancy or at immediate postpartum. Psychological support could then be provided to the mother, with a referral to specialized treatment if necessary. In this manner, breastfeeding and optimal care for the newborn in its first days of life could be guaranteed.

Our investigation will appeal to an international and interdisciplinary audience, given the high worldwide prevalence of unplanned pregnancy and psychological disorders; and the implications for postpartum screening and treatment worldwide.

\section{Conclusion}

Women with unplanned pregnancies have more frequency of psycho-emotional disorders. It is suggested screening not only for postpartum depression, but also for more comprehensive psycho-emotional disorders in women with unplanned pregnancies. Then early psychological support could be provided to the mother.

\section{References}

[1] Santelli J, Rochat R, Hatfield-Timajchy K, Colley GB, Curtis K, Cabral R. Hirsch JS, Schieve L. The measurement and meaning of unintended pregnancy. Perspectives on Sexual and Reproductive Health 2003; 35: 94-101

[2] Lifflander A, Gaydos LM, Hogue CJ. Circumstances of pregnancy: low income women in Georgia describe the difference between planned and unplanned pregnancies. Matern Child Health J 2007; 11: 81-89.

[3] Benso R. Rekindling efforts to prevent unplanned pregnancy: a matter of equity and common sense. Guttmacher Policy Review 2006; 9: 1-7.

[4] Wellings K, Jones KG, Mercer CH, Tanton C, Clifton S, Datta J, Copas AJ, Erens B, Gibson LJ, Macdowall W, Sonnenberg P, Phelps A, Johnson AM. The prevalence of unplanned pregnancy and associated factors in Britain: findings from the third National Survey of Sexual Attitudes and Lifestyles (Natsal-3). Lancet 2013; 382: 1807-1816.

[5] Eastwood JG, Phung H, Barnett B. Postnatal depression and socio-demographic risk: factors associated with Edinburgh Depression Scale scores in a metropolitan area of New South Wales, Australia. Australian New Zealand Journal of Psychiatry 2011; 45: 1040-1046.

[6] Hoga LA, Manganiello A. Male behaviors towards unplanned pregnancy: experiences of Brazilian low-income women. International Nursing Review 2007; 54: 346-353.

[7] Gamboa G, Lozano L. Unplanned pregnancies affect the lives of millions of Latin women. Accessed January 22, 2013. Available at

http://www.terra.com/salud/articulo/html/sal1108.htm

[8] Van den Bergh BR, Mennes M, Stevens V. ADHD deficit as measured in adolescent boys with a continuous performance task is related to antenatal maternal anxiety. Pediatric Research 2006; 59: 78-82.

[9] Sayil M, Güre A, Uçanok Z. First time mothers' anxiety and depressive symptoms across the transition to motherhood: associations with maternal and environmental characteristics. Women Health 2006; 44: 61-77.

[10] Butcher JN, Dahlstrom WG, Graham JR, Tellegen A, Kaemmer B. The Minnesota Multiphasic Personality Inventory-2 (MMPI-2): Manual for administration and scoring. Minneapolis, MN: University of Minnesota Press 1989.

[11] D’Orazio LM, Meverowitz BE, Korst LM, Romero R, Goodwin TM. Evidence against a link between hyperemesis gravidarum and personality characteristics from an ethnically diverse sample of pregnant women: a pilot study. J Womens Health (Larchmt) 2011; 20: 137-144.

[12] Hintze J. Number Cruncher Statistical System. Kaysville, Utah: Statistical Software, 2005. Accessed December 6, 2012.Available at http://www.ncss.com.

[13] Duran MM, Sauceda GJM, Lartigue T. Physiological and 
emotional changes during pregnancy and behavior of the fetus. Perinatología y Reproducción Humana 2008; 22: $5-14$.

[14] Dubey C, Gupta N, Bhasin S, Muthal RA, Arora R. Prevalence and associated risk factors for postpartum depression in women attending a tertiary hospital Delhi, India. International Journal Society of Psychiatry 2012; 58: 577-680.

[15] Rosenfeld JA, Everett KD. Factors related to planned and unplanned pregnancies. Journal of Family Practice 1996, 43, 161-166.

[16] Bennett IM, Culhane JF, McCollum KF, Elo IT. Unintended rapid repeat pregnancy and low education status: any role for depression and contraceptive use? American Journal of Obstetrics and Gynecology 2006; 194: 749-754.

[17] Gao W, Paterson J, Carter S, Iusitini L. Intimate partner violence and unplanned pregnancy in the Pacific Islands
Families Study. International Journal of Gynecology and Obstetrics 2008; 100: 109-115.

[18] Nakku JE, Nakasi G, Mirembe F. Postpartum major depression at six weeks in primary health care: prevalence and associated factors. African Health Sciences 2006; 6: 207-214.

[19] Boyce P, Hickey A. Psychosocial risk factors to major depression after childbirth. Social Psychiatry and Psychiatric Epidemiology 2005; 40: 605-612.

[20] Bener A, Gerber L, Sheikh J. Prevalence of psychiatric disorders and associated risk factors in women during their postpartum period: a major public health problem and global comparison. Int J Womens Health 2012; 4: 191-200.

[21] Iranfar S, Shakeri J, Ranjbar M, NazhadJafar P, Razaie M. Is unintended pregnancy a risk factor for depression in Iranian women? Eastern Mediterranean Health Journal 2005; 11: 618-624. 